\title{
Simulation of 2D surface flow in open channel using explicit finite difference method
}

\author{
Shakeel Ahmed Kamboh \\ Department of Mathematics \& Statistics \\ Faculty of Science, QUEST \\ 67450 Nawabshah, Pakistan \\ e-mail: shakeel.maths@yahoo.com
}

\author{
Izzatul Nabila bt Sarbini, Jane Labadin \\ Department of Computational Science \& Mathematics \\ Faculty of Computer Science \& \\ Information Technology, UNIMAS \\ e-mail: sinabila@fit.unimas.my \\ e-mail: ljane@fit.unimas.my
}

\begin{abstract}
D surface flow models are useful to understand and predict the flow through breach, over a dyke or over the floodplains. This paper is aimed at the surface flows to study the behavior of flood waves. The open channel water flow in drains and rivers is considered in view of the fact that such flows are the source of flash flood. In order to predict and simulate the flood behavior a mathematical model with the initial and boundary conditions is established using 2D Saint-Venant partial differential equations. Next, the corresponding model is discretized by using the explicit finite difference method and implemented on MATLAB. For the testing and implementation purpose a simple rectangular flow channel is considered. The output parameters like height or depth of water, the fluid velocity and the volumetric flow rate are simulated numerically and visualized for the different time steps. The initial simulation results are useful to understand and predict the flood behavior at different locations of flow channel at specific time steps and can be helpful in early flood warning systems.
\end{abstract}

Keywords-surface flow; open channel; Saint-Venant equations; shallow water equations

\section{INTRODUCTION AND BACKGROUND}

Flood is the most frequently occurring natural disaster that not only affects the large population and the agricultural lands but also results in the loss of human life and damage of infrastructure [1]. Increased runoff rates due to urbanization, prolonged rainfall and inadequate river capacity are some of the major causes of the flood occurrences. It is very important to understand the behavior of water flow in the flow channels for early flood disaster management and also for saving human life. In early days, the methods used to predict the daily flows were limited to forecast only at the cross sections of flow channels and some times appeared to be unsuitable [2]. The mathematical models are quite useful to govern the fluid flow of different phenomenon including the surface flow, subsurface flow and coupled flows. In this regards, various studies have been done for the modeling of flood events at floodplains. For the one dimensional applications the flood wave propagation dynamic equations were proposed by [3] and are commonly known as Saint-Venant (SV) equations. These equations are widely used to predict the surface flow parameters such as velocity, depth or height and the flow rate. For the two dimensional surface flow the SV equations are derived from the Navier-Stokes equations [4] and often referred to as Shallow Water (SW) equations. The derivation of SW equations and some of its variants can be found in [5]. Due to the non-linear nature of SV equations, the analytical solution is quite difficult. Therefore, the different numerical techniques have been proposed to obtain the surface flow simulations [6]. A numerical method based on the MacCormack finite difference scheme for simulating discontinuous two-dimensional overland shallow flow with spatially variable for micro topography was developed by [7]. The method is useful to simulate the rainfall-driven flash floods, flow in ephemeral stream channels, and tidal flat and wetland circulation. Another method based on an explicit finite difference scheme for micro topography was proposed by [8] which are useful for coupling overland surface flow and the infiltration processes during complex rainfall on natural slopes. [9] Presented explicit and implicit finite volume method schemes of the Roe type to model extreme unsteady, rapidly varied, open channel surface flow described by the SV equations. The numerical solution schemes devised by [9] are useful to simulate the transient flow, when discontinuities such as hydraulic jumps and bores exist. To analyze the depth of water surface in flood channel [10] proposed a numerical method to discretize the 1D SV equations. The results revealed that for the same value of roughness coefficient the smaller values of the bed slope of channel tend to high depth of water at specific time step. For simulating flood wave in the natural rivers [11] used SV equations and presented the numerical results of two different finite difference methods based on Preissmann and Lax diffusive schemes. They compared the hydrographs of both the methods with commercial simulation program HEC-RAS for the flow discharge and depth of water at $15 \mathrm{~km}$ upstream end and at the down stream end. It was concluded by [11] that the results obtained by Preissmann and Lax diffusive schemes are reasonably closed to each other but there was a poor agreement of these methods with the output results produced by HEC-RAS. One of the reasons of such disagreement in the 